\title{
Low-Frequency Noise in Graphene Tunnel Junctions
}

\author{
Paweł Puczkarski, ${ }^{\dagger}{ }^{\circ}$ Qingqing Wu, ${ }^{\ddagger}$ Hatef Sadeghi, ${ }^{\ddagger}$ (๑) Songjun Hou, ${ }^{\ddagger}$ Amin Karimi, ${ }^{\dagger}$ Yuewen Sheng, $^{\dagger}$

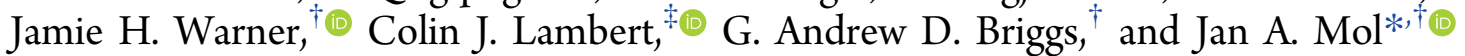

$\dagger$ Department of Materials, University of Oxford, 16 Parks Road, Oxford OX1 3PH, United Kingdom

${ }^{\ddagger}$ Department of Physics, Lancaster University, Bailrigg, Lancaster LA1 4YB, United Kingdom

\section{Supporting Information}

\begin{abstract}
Graphene tunnel junctions are a promising experimental platform for single molecule electronics and biosensing. Ultimately their noise properties will play a critical role in developing these applications. Here we report a study of electrical noise in graphene tunnel junctions fabricated through feedbackcontrolled electroburning. We observe random telegraph signals characterized by a Lorentzian noise spectrum at cryogenic temperatures $(77 \mathrm{~K})$ and a $1 / f$ noise spectrum at room temperature. To gain insight into the origin of these noise features, we introduce a theoretical model that couples a quantum mechanical tunnel barrier to one or more classical fluctuators. The fluctuators are identified as charge traps in the underlying dielectric, which through random fluctuations in

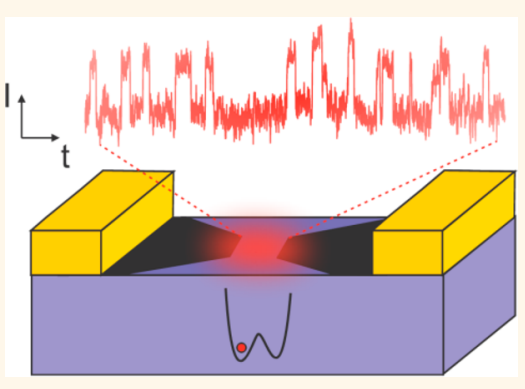
their occupation introduce time-dependent modulations in the electrostatic environment that shift the potential barrier of the junction. Analysis of the experimental results and the tight-binding model indicate that the random trap occupation is governed by Poisson statistics. In the 35 devices measured at room temperature, we observe a $20-60 \%$ time-dependent variance of the current, which can be attributed to a relative potential barrier shift of between $6 \%$ and $10 \%$. In 10 devices measured at $77 \mathrm{~K}$, we observe a $10 \%$ time-dependent variance of the current, which can be attributed to a relative potential barrier shift of between $3 \%$ and $4 \%$. Our measurements reveal a high sensitivity of the graphene tunnel junctions to their local electrostatic environment, with observable features of intertrap Coulomb interactions in the distribution of current switching amplitudes.
\end{abstract}

KEYWORDS: graphene, tunnel junctions, low frequency noise, random telegraph noise, charge traps

raphene tunnel junctions provide a two-dimensional platform for probing individual molecules. Recent experiments have demonstrated charge transport through single molecules that were firmly anchored between a pair of graphene electrodes via $\pi-\pi$ stacking $^{1-3}$ or covalent bonding. ${ }^{4-8}$ Moreover, graphene tunnel junctions have been proposed as candidate systems for molecular sensing, in particular for sequencing DNA molecules as they translocate through the gap. ${ }^{9}$ These devices rely on the unique material properties of graphene: its two-dimensional nature, zeroenergy bandgap, and semimetallic type conductance. ${ }^{10}$ The same properties also make graphene unique in the context of low-frequency noise, ${ }^{11}$ with both carrier fluctuations and mobility fluctuations ${ }^{12-29}$ playing an important role. ${ }^{30}$ Whether graphene retains its favorable noise properties when structured into an $\sim 1 \mathrm{~nm}$ wide nanogap becomes particularly pertinent for applications that require a large signal-to-noise ratio, such as DNA sequencing. ${ }^{31-34}$

Low-frequency $1 / f$ noise or "flicker" noise is ubiquitous in nanoscale electronic systems, leading to prominent current fluctuations in semiconductor devices, ${ }^{35-39}$ tunnel junctions, $^{40-43}$ and nanopores. ${ }^{44-49}$ While the physical mecha- nisms that generate these fluctuations may vary and are often not known, it is generally accepted that $1 / f$ noise is the result of a distribution of nonidentical random telegraph signals (RTSs). ${ }^{11,35,36,39,50}$ These RTSs each have a Lorentzian noise power spectral density, the superposition of which results in a $1 / f$ power spectral density. The emergence of $1 / f$ noise from a distribution of nonidentical fluctuators was first described by McWhorter ${ }^{35,51}$ in the context of interface traps in metaloxide-semiconductor field-effect transistors (MOSFETs), where trapping and detrapping of charge results in fluctuations in the number of charge carriers in the semiconductor channel. ${ }^{36,37,39}$

RTSs have been observed experimentally in carbon nanotubes and have been predicted in graphene nanoribbons. These RTSs originate from the sensitivity of carbon nanotubes and graphene nanoribbons to a limited number of fluctuators in a small contact area. ${ }^{52,53}$ In micrometer-scale graphene channels, relatively low noise amplitudes have been reported

Received: June 21, 2018

Accepted: August 16, 2018

Published: August 16, 2018 

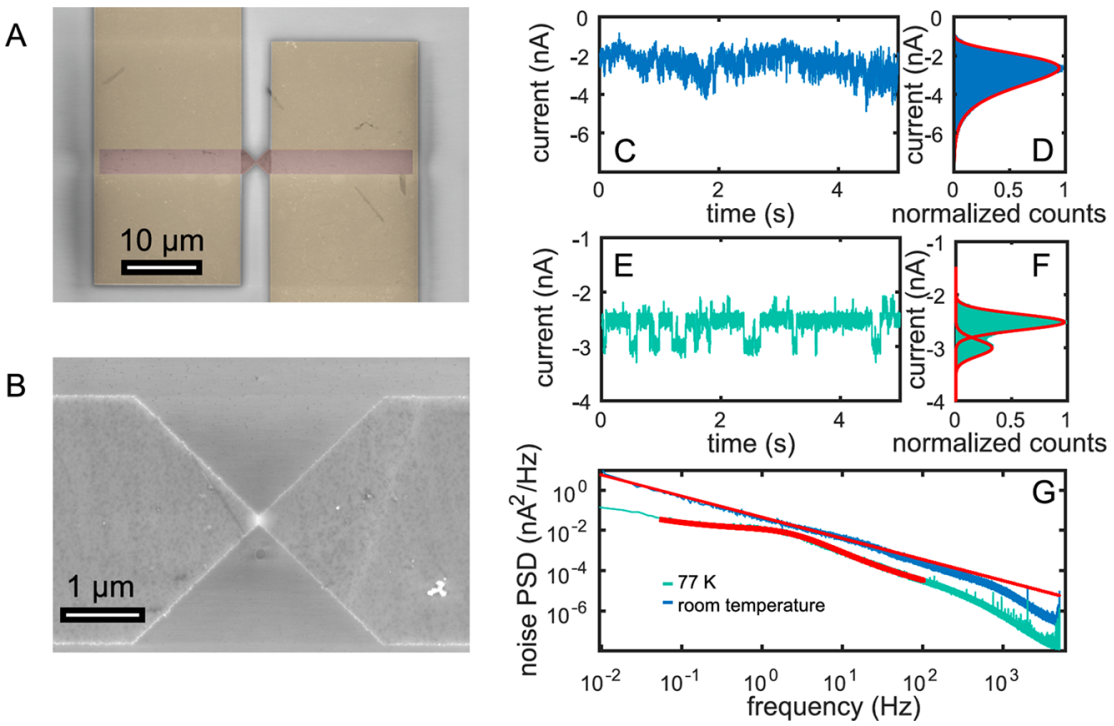

Figure 1. SEM images of (A) gold electrodes with a graphene device with a constriction in the middle, and a zoom-in image of (B) constriction with the localized tunnel junction. Fluctuations in tunneling current in graphene tunnel junctions and resulting noise spectra: (C) Nonspecific fluctuations in tunneling current at room temperature and (D) The corresponding log-normal distribution of current values. (E) RTS in $I-t$ traces and (F) bimodal current distribution with two Gaussian peaks upon cooling the device to $77 \mathrm{~K}$. (G) Current noise PSD measured in graphene tunnel junctions has $1 / f$ form at room temperature and Lorentzian form at $77 \mathrm{~K}$, with lower overall noise level.

comparable to those found in state-of-the-art silicon transistors. ${ }^{19}$ When the width of a graphene nanoribbon is reduced below $100 \mathrm{~nm}$, the noise can increase by $2-3$ orders of magnitude. ${ }^{54}$ Until now, RTSs have not been reported in graphene nanogaps. In the case of tunnel junctions, fluctuations in the electrostatic environment ${ }^{55-57}$ and mechanical $^{58-61}$ instabilities will lead to noise in the tunnel current through modulation of the transmission function. $^{40,41,62}$

Here, we investigate the noise properties of nanometer-sized graphene tunnel junctions and present a theoretical description of RTSs and the emergence of $1 / f$ noise, resulting from a quantum mechanical system coupled to either a single fluctuator or a distribution of classical fluctuators, respectively. Graphene tunnel junctions are fabricated using feedbackcontrolled electroburning (see Methods) and measured at room temperature and at $77 \mathrm{~K}$. The current is sampled at 100 $\mathrm{kHz}$ with a low-pass filter with a cutoff frequency of 1 or 10 $\mathrm{kHz}$. The mean current depends exponentially on the applied bias voltage and is well described by the Simmons model. ${ }^{63}$ Fitting the $I-V$ curves to the Simmons model yields an average gap size of $\sim 1.5 \pm 0.2 \mathrm{~nm}$ (See Methods and the Supporting Information (SI) for further details concerning statistics of gap sizes and the method of their measurement), consistent with electroburnt gaps reported in earlier studies. $1,64,65$

\section{RESULTS AND DISCUSSION}

Current Fluctuations in Graphene Tunnel Junctions. Our devices consist of a graphene ribbon patterned on top of a pair of gold electrodes (see Figure 1A). The graphene ribbon has a $200 \mathrm{~nm}$ constriction, which allows for the localized electroburning of a tunnel junction between two parts of the graphene ribbon (see Figure 1B). Figure 1C and E shows typical current-time $(I-t)$ traces measured for a graphene tunnel junction at room temperature and at $77 \mathrm{~K}$, respectively. The room temperature $I-t$ trace (Figure 1C) shows characteristic flicker noise behavior, where, like the light of a flickering candle, the signal has a wandering baseline as the high frequency noise rides on a low frequency component. By contrast, the $77 \mathrm{~K} \mathrm{I}-t$ trace (Figure 1E) predominantly fluctuates between two levels, indicating that a single two-level fluctuator dominates the noise. The observed current fluctuations are also evident from the bimodal Gaussian distribution of current values (Figure $1 \mathrm{~F}$ ) and can be measured for up to $6 \mathrm{~h}$. (see the SI) A histogram of the room temperature current in graphene tunnel junctions (Figure 1D) reveals a distinct log-normal distribution of the current values and gives a first hint at the physical mechanism behind the $1 / f$ noise. A simplified formulation of the Simmons model gives the tunnel current ${ }^{63}$

$$
I \propto \int n(E) \mathcal{T}(E) \mathrm{d} E
$$

where $n(E)$ is the carrier density and the probability that an electron can cross a tunnel barrier with width $d$ and height $\varphi$ is given by the $\mathrm{WKB}$-approximation:

$$
\mathcal{T}(E)=\mathrm{e}^{-d \sqrt{2 m(\varphi-E) / \hbar^{2}}}
$$

If the number of charge carriers were to fluctuate according to a normal distribution, this would result in a normal distribution of the current values. However, if the barrier height or width fluctuates according to a normal distribution this results in the observed log-normal distribution of the current, due to the exponential dependence of the transmission function $\mathcal{T}(E)$.

Noise Power Spectral Densities. By comparing the noise power spectral density (PSD) $S_{I}(f)$ of the tunnel junction at room temperature and at $77 \mathrm{~K}$ (Figure $1 \mathrm{G}$ ), we find that $S_{I}(f)$ at $T=293 \mathrm{~K}$ is well described by $\frac{A}{f^{\gamma}}$, whereas $S_{I}(f)$ at $T=77 \mathrm{~K}$ shows a distinct corner at $f=7.4 \mathrm{~Hz}$ superimposed onto a linear slope $\frac{A}{f^{\gamma}}$. Since the density of thermally activated fluctuators is typically not constant in space and activation energy, fluctuations can be dominated by a single fluctuator 
within a given spectral window when the temperature is sufficiently reduced. ${ }^{36-38}$ The noise PSD of a single two-level fluctuator is given by ${ }^{66,67}$

$$
S_{I}(f)=\frac{2 \Delta I^{2} \tau}{4+(2 \pi f \tau)^{2}}
$$

where $\Delta I$ is the change in the current induced by the fluctuator and $\tau$ the mean dwell time of the fluctuator. In the case of simple RTSs between up and down states, $\tau$ is an average value of the dwell time of the up $\left(\tau_{\text {up }}\right)$ and down $\left(\tau_{\text {down }}\right)$ level, $\frac{1}{\tau}=\frac{1}{\tau_{\text {up }}}+\frac{1}{\tau_{\text {down }}} \cdot{ }^{62}$ These RTSs are universally observed for all graphene tunnel junctions at $77 \mathrm{~K}$ temperature. The $I-t$ traces measured for a different device are separated into individual current levels by a change point detection method (Figure 2A).
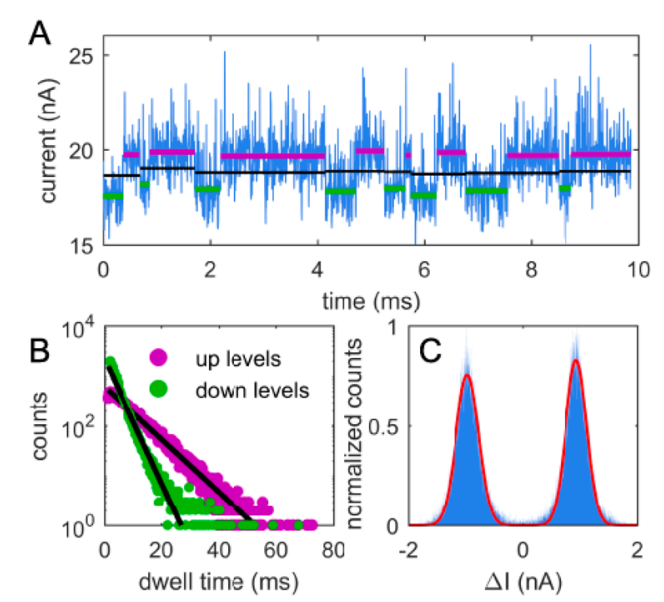

Figure 2. Analysis of RTSs in graphene tunnel junctions measured at $77 \mathrm{~K}$. (A) Fragment of $I-t$ trace with marked separate up and down levels and local baseline for pairs of switching levels (black). (B) Distribution of the dwell times for both levels with fits to Poisson distributions. (C) Distribution of current level values for both levels, fitted with a Gaussian distribution.

As expected for the RTS, the dwell time for both levels follows a Poisson distribution $P(\tau) \sim \exp \left(-\frac{\tau}{\langle\tau\rangle}\right)^{40,68,69}$ (Figure 2B). A fit to the Poisson model enables us to obtain the mean dwell time values, $\tau_{\text {up }}=13.0 \mathrm{~ms}$ and $\tau_{\text {down }}=4.3 \mathrm{~ms}$. The separation of $I-t$ traces into separate levels allows for a closer examination of the current step values. The consecutive up and down levels are grouped into pairs and the mean value of each pair $\bar{I}=\left(I_{\text {up }}\right.$ $\left.+I_{\text {down }}\right) / 2$ is used as a reference level to calculate the current step height $\Delta I_{\text {up } / \text { down }}=I_{\text {up } / \text { down }}-\bar{I}$. The distribution of $\Delta I$ for up and down levels (Figure 2C) shows a good separation between the current levels, which are centered at the mean values and can be fitted with a Gaussian distribution.

If the fluctuations are thermally activated, the process follows an Arrhenius law $\tau^{-1}=\tau_{0}{ }^{-1} \mathrm{e}^{-E_{a} / k_{\mathrm{B}} T}$, and reducing the temperature will decrease the corner frequency $\tau^{-1} \cdot{ }^{35,36,39,69} \mathrm{By}$ changing the temperature we therefore sample a different subset of the collection of nonidentical RTSs. The fact that we observe a single dominant RTS at $77 \mathrm{~K}$ indicates that at this temperature we are sampling a smaller number of RTSs. Similar temperature dependent behavior has previously been reported in metal-oxide-semiconductor devices, where it is attributed to the energy-dependent interface trap density in the oxide layer. $^{36-39,70}$
The dependence of the amplitude and dwell time of the RTS on applied voltage and mean current is presented in Figure 3.
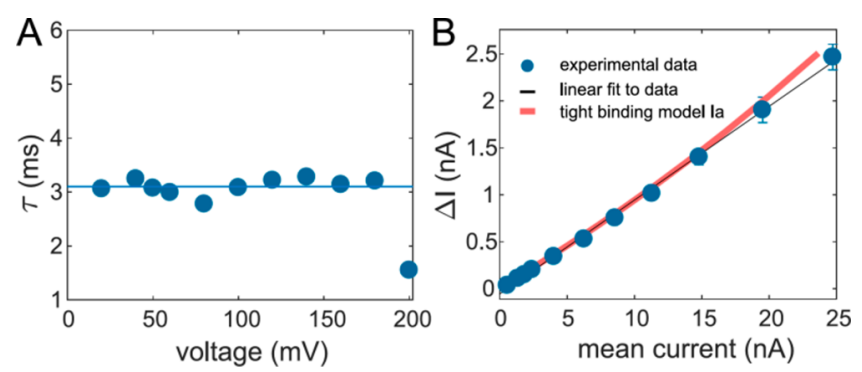

Figure 3. Scaling of RTS parameters with voltage bias and tunneling current. (A) Dependence of the mean dwell time $\tau$ on applied voltage. Horizontal line shows $\tau=3.1 \mathrm{~ms}$ reference level. (B) Dependence of the measured $\Delta I$ amplitude, and $\Delta I$ amplitudes obtained from tight binding model Ia, on the mean tunneling current.

The dwell time distribution shows no meaningful trend within the experimental error bars with increasing voltage (Figure $3 \mathrm{~A})$. There is an approximately linear increase of the RTS amplitude $\Delta I$ with increasing mean tunneling current (Figure 3B). This indicates that the tunneling current does not drive the observed fluctuations in conductance, but that these fluctuations exist independently of the current and the current is merely a readout method of the independent fluctuations. ${ }^{39}$ The same approximately linear relationship for low voltages is obtained in the tight binding model presented below, where the environmental fluctuators driving the tunnel barrier are independent of the current or applied voltage (Figures $3 \mathrm{~B}$ and SI2).

To characterize the $1 / f$ noise amplitude, we compare the normalized noise power spectral density $S_{I}(f) / I^{2}$ for 35 devices in Figure 4. The noise spectra recorded for several voltage values show that the $1 / f$ noise profile is present independent of the applied voltage and increasing voltage does not induce Lorentzian noise spectrum at room temperature (Figure 4A). We find that the exponent $\gamma=1 \pm 0.2$ (Figure 4B) does not
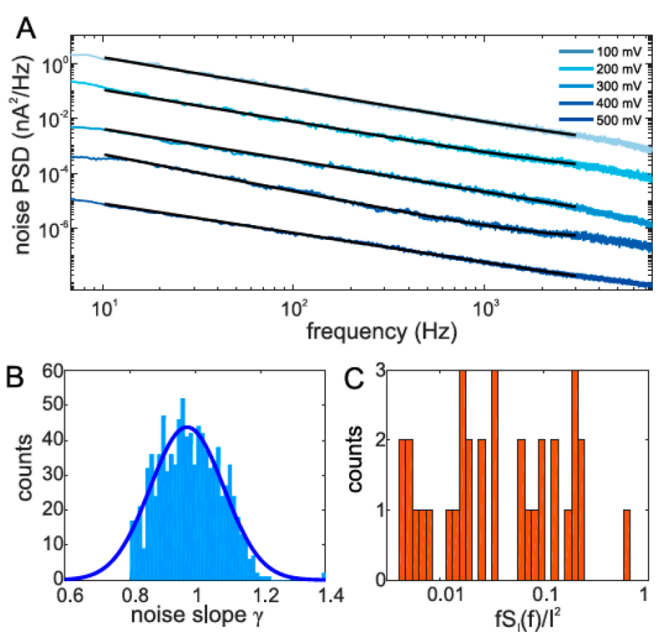

Figure 4. $1 / f$ noise in graphene tunnel junctions at room temperature. (A) Noise spectra for several bias values with fitted $1 / f$ curves (black). (B) Distribution of $\gamma$ slopes fitted with Gaussian function. (C) Distribution of normalized $f S_{I}(f) / I^{2}$ noise amplitude for 35 measured graphene tunnel junctions. 
depend on the tunneling current (Figure SI14). Deviations from a $1 / f$ noise profile are typically attributed to variations in the distribution of the RTSs, ${ }^{35,36,39,50}$ and the $\gamma$ values obtained in our graphene tunnel junctions are in the same range as values obtained for silicon devices, ${ }^{35-39}$ tunnel junctions, ${ }^{40-43}$ and nanopores. ${ }^{44-49} \mathrm{We}$ also find that $S_{I}(f) / I^{2}$ measured for the same device at different bias voltages remains unchanged, indicating that the noise is not driven by the current and that $\Delta I \propto I$.

More surprising are the values for the normalized noise amplitude, or pseudo-Hooge parameter, $\alpha=f S_{I}(f) / I^{2}$, which ranges from $\log \alpha=-3$ to 0 (Figure $4 \mathrm{C}$ ). These values are $7-9$ orders of magnitude larger than those reported in micrometersized graphene channels, ${ }^{13,16,18,19,71,72}$ and 2 to 3 orders of magnitude higher than the normalized noise amplitude measured in graphene nanopores of comparable size to our tunnel junctions. ${ }^{48,73}$ This may be attributed to the extreme sensitivity of the tunnel current (compared to for example the ionic current in nanopores) to environmental fluctuations. When we compare the noise characteristics of our devices to those reported for MOSFET-type device of similar dimensions we find that pseudo-Hooge parameters in silicon devices are at least 2 orders of magnitude lower, ${ }^{74-78}$ which is likely due to the highly optimized semiconductor fabrication processes that minimize the number of interface traps in the oxide. ${ }^{77,79}$ When we compare our devices to CNT transistors on thermally grown $\mathrm{SiO}_{2},{ }^{80-84}$ we find similar noise values to our devices. In the remainder of this work we shall present a theoretical model explaining the sensitivity of graphene tunnel junctions to fluctuations in their electrostatic environment, and identify the potential mechanisms for causing these fluctuations.

Tight Binding Model of a Tunnel Junction. One possible origin of the observed RTS and Lorentzian noise spectrum is the presence of charge traps distributed in the substrate underlying graphene tunnel junctions. By changing their charge state between empty and occupied, traps alter the electrostatic environment of the junction, which may lead to the shift of the potential barrier in the junction with respect to the Fermi level of graphene electrodes. To gauge the effect of fluctuations in the charge trap occupation on the current through the tunnel junction we employ a simple onedimensional Hückel tight binding model. The model consists of a quantum tunnel barrier driven by the classical environment. The tunnel barrier is modeled as a scattering region containing $N$ quantum levels, connected to two semi-infinite electrodes (Figure 5). The barrier is coupled to the classical fluctuating environment, which is represented by one or more generalized coordinates $x_{i}$ corresponding to charge traps. The modeled coupling between the quantum system and environmental classical system yields a simple linear $\varepsilon \sim x$ relationship.

The aim of the model is to understand how different parameters describing the classical environment affect the changes in tunneling signal and in particular to estimate the magnitude of potential barrier fluctuations which can give rise to the observed current features. We investigate two models representing four limiting cases. Model I describes the case where five quantum levels in the scattering region are driven synchronously $\left\{\varepsilon_{1}=\varepsilon_{2}=\ldots=\varepsilon_{5}=\varepsilon\right\}$ by the collective effect of $N$ traps $\left\{x_{1}, x_{2}, \cdots, x_{N}\right\}$, such that $\varepsilon=a_{1}+\sum_{n=1}^{N} x_{i} / b_{1}$. For model Ia, $N=1$, whereas for model Ib $N=5$ (Figure 5). In the SI, we consider two variants of a second model in which $N$ fluctuators $\left\{x_{1}, x_{2}, \ldots, x_{N}\right\}$, couple individually to $N$ quantum

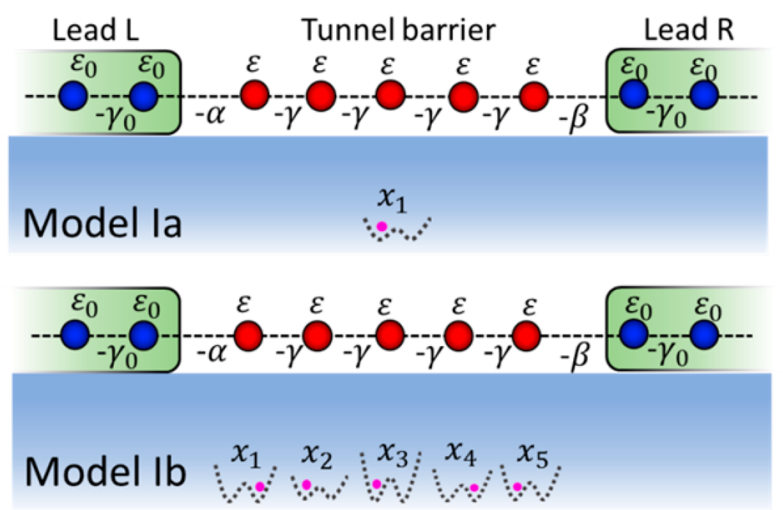

Figure 5. Tight binding model with individual quantum levels driven by collective traps effect. The on-site energies of the left and right electrodes (blue dots) are denoted $\varepsilon_{0}=0$. The tunnel barrier in the scattering region is formed by individual quantum levels (red dots) with on-site energies $\varepsilon_{i}$ which are allowed to fluctuate due to the interaction with the environmental charge $x_{i}$. The hopping integrals $\gamma_{0}, \gamma$ are all set to unity and $\alpha=\beta=0.35$ represent the weaker coupling between the electrodes and scattering region.

levels in a one-to-one manner $\varepsilon_{i}=a_{2}+\frac{x_{i}}{b_{2}}$. In model IIa, $N=$ 1 , whereas in model IIb $N=5$. Models Ia and IIa with fluctuations driven by a single fluctuator correspond to the measurements at $77 \mathrm{~K}$, while models $\mathrm{Ib}$ and IIb with multiple fluctuators influencing the barrier represent the measurements at room temperature with more thermally excited charge traps allowed to fluctuate. Models IIa and IIb, where fluctuators independently couple to individual sites in the barrier, correspond to local perturbations of the barrier by nearby interface traps, whereas models $\mathrm{Ia}$ and $\mathrm{Ib}$ correspond to traps that are far from the tunnel barrier. Considering the size of the tunnel junction $(\sim 1 \mathrm{~nm})$ and expected spacing of charge traps in the substrate $(\sim 10 \mathrm{~nm})^{21,85}$ the latter case is more realistic. The time dependence of the fluctuators is described by a Langevin equation (details in the SI).

Fluctuations in the Tunnel Barrier. In the tight binding model, the height of the resulting tunnel barrier $u$ between two leads is the difference between the Fermi level (black dashed line in Figure 6A) and the mean value of the lowest eigenvalue of the scattering region, corresponding to the nearest
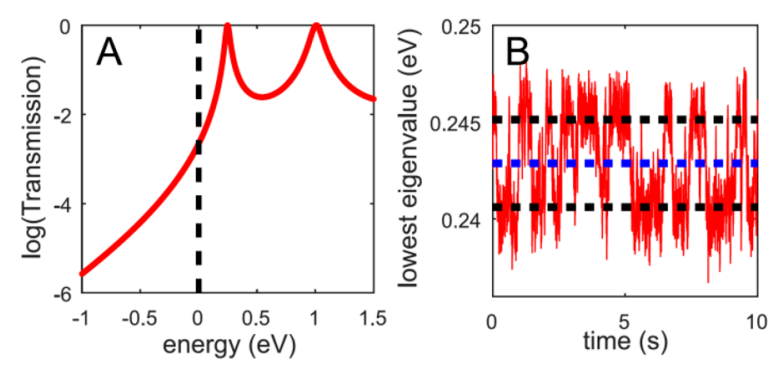

Figure 6. Fluctuations of eigenvalues leading to the alteration of transmission of the junction. (A) Transmission spectrum at one specific time. (B) Lowest eigenvalue trace among the five eigenvalues. Blue dashed baseline indicates the mean tunnel barrier height $u$ referred to the Fermi level of the whole device, $E_{\mathrm{F}}$ $=0$. Two black dashed baselines above and below are the mean values for two fluctuating levels spaced by $\Delta u$. For these simulations, $a_{1}=1.975, b_{1}=150$. 
transmission resonance (at $0.25 \mathrm{eV}$ in Figure 6A). For the model Ia, because of the influence of the generalized environmental coordinate $x_{1}$, the lowest eigenvalue $E_{1}$ fluctuates over time with a mean value $u$ (blue dashed line in Figure 6B) and mean upper and lower values $\left(u+\frac{\Delta u}{2}, u-\frac{\Delta u}{2}\right)$ (black dashed lines in Figure 6B).

Current Fluctuations in the Tight Binding Model. The I- $t$ traces for models Ia (Figure 7C) and IIa (Figure SI5C)
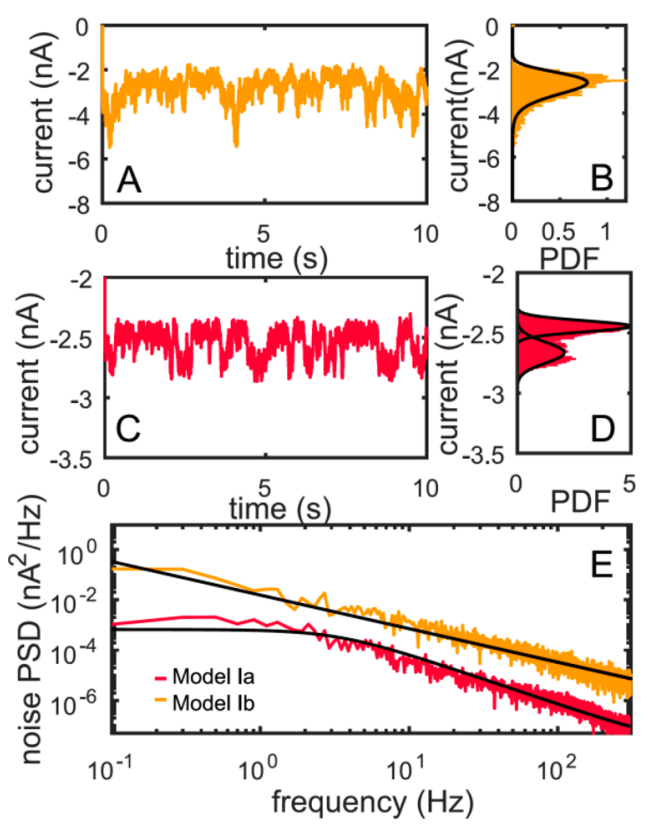

Figure 7. Features of current traces and noise PSD corresponding to the tight binding model I. (A) $I-t$ trace and (B) log-normal current distribution for the model $\mathrm{Ib}$. The relationship between $\varepsilon$ and $x_{i}$ is $\varepsilon=a+\sum_{n=1}^{5} x_{i} / b$ and $\left\{c_{i}\right\}=\{0.4,0.8,1.2,1.8,2.5\}$. (C) $I-t$ trace and (D) current histogram for the model Ia $(c=0.4)$. (E) Noise PSD following Lorentzian trend for the model Ia and $1 / f$ trend for the model Ib. For these simulations, $a=1.975, b=150$.

show a distinct RTS, in contrast to the $I-t$ ftraces for the case of Ib (Figure 7A) and IIb (Figure SI5A), which have the characteristic wandering baseline associated with flicker noise. The current histograms for models Ia (Figure 7D) and IIa (Figure SI5D) contain two Gaussian peaks, while the histograms for models Ib (Figure 7B) and IIb (Figure SI5B) have the log-normal distribution that was observed in our room temperature experiments. The noise spectra for the single-trap models (Ia and IIa) have a Lorentzian frequency dependence and as more environmental fluctuators are activated in the models $\mathrm{Ib}$ and IIb, a $1 / f$ noise spectrum emerges, corresponding to the thermal activation of multiple RTSs at room temperature. We find that the slope varies between 0.9-1.3, when tuning the tunnel barrier height $u$ shown in blue dashed line in Figure 6B, which agrees with measured sample to sample variations (see more details in Figures SI3 and SI6).

The tight binding model also reproduces scaling features of the experimental data showing an exponential increase of the amplitude $\Delta I$ of RTSs as a function of bias voltage (Figure SI2A). This feature arises, because the Fermi level is located in the exponential tail of the transmission coefficient $\mathcal{T}(E)$, which is controlled by the lowest eigenvalue. The model also shows the linear increase of the amplitude $\Delta I$ as a function of the mean current in agreement with the experimental data (Figure 3B). The slope of the $\Delta I \sim I$ dependence is 0.1 which is also in qualitative agreement with the experimental results. This qualitative agreement corresponds to a relative barrier-height fluctuation of $\frac{\Delta u}{u}=0.028$ for the model Ia and $\frac{\Delta u}{u}=0.035$ for the model IIa. Experimentally, potential shifts of this order can be induced by switching of an electron from a charge trap located at distance of a few $\mathrm{nm}$ from the junction to another one that is a few nm further away (details in the SI, Figure SI13). The tight binding model also shows that five traps controlling transport through the tunnel junction are sufficient to produce $1 / f$ noise over a four-decade frequency range, consistent with other reports. ${ }^{50}$ The room temperature models $\mathrm{Ib}$ and IIb also show that the normal distribution of the potential shifts $\Delta u$ results in the log-normal current distribution. The width $\Delta u=\left\langle\left(E_{1}-u\right)^{2}\right\rangle^{1 / 2}$ of the modeled potential distributions is equal to $\frac{\Delta u}{u}=0.057$ and $\frac{\Delta u}{u}=0.094$ for models $\mathrm{Ib}$ and $\mathrm{IIb}$, respectively. All four models confirm that noise is not driven by current, because the environmental fluctuators are independent of the applied voltage or current.

Potential fluctuations. To estimate the potential shift due to the fluctuations in the charge trap occupation at room temperature, we assume that pairs of filled and unfilled charge traps are represented by electric dipoles of charge $\pm e$ spaced by a distance $d=10 \mathrm{~nm}$, corresponding to a typical trap concentration $\rho=1 \times 10^{18} \mathrm{~cm}^{-3} \cdot{ }^{72,85}$ The dipoles are located in the nodes of cubic lattice of total size $2000 \mathrm{~nm} \times 2000 \mathrm{~nm} \times$ $2000 \mathrm{~nm}$, with a $20 \mathrm{~nm}$ lattice constant, which gives the correct value of the charge trap density, assuming a $10 \mathrm{~nm}$ intertrap spacing. Variability in the potential is introduced by allowing all the dipoles to take a random orientation $\Theta$ with respect to the axis connecting the center of the dipole and the center of the junction (Figure $8 \mathrm{~A}$ ). Each of the dipoles at distance $R$ gives the potential contribution $V_{i}(r, \Theta)=\frac{q d \cos \Theta}{4 \pi \varepsilon_{0} \varepsilon_{\mathrm{r}} r^{2}}$, where $\varepsilon_{0}$ is the vacuum permittivity, $\varepsilon_{\mathrm{r}}=3.9$ is the relative permittivity of $\mathrm{SiO}_{2}$, and $q$ is the elementary charge. The net potential of the junction resulting from the dipole lattice as a function of the radius $R$ is calculated as a sum of potential contributions for all dipoles at distance $r<R, V_{R}=\sum_{r=0}^{r<R} V_{i}\left(r_{i}, \Theta_{i}\right)$. In Figure 8B, we plot, for example, the cumulative net potential as a function of radius $R$ for nine randomly chosen dipole lattice distributions (with different random orientations $\Theta_{i}$ of dipoles at a given lattice node). Only the dipoles nearest to the junction significantly affect the potential. Charge traps at large distances $R>400 \mathrm{~nm}$ do not induce large changes in the net potential, due to the decreasing contribution from each dipole and the increasing number of randomly oriented dipoles. Therefore, the potential value summed for all traps with $r \leq$ $1000 \mathrm{~nm}$ is taken as the final potential value.

In order to simulate the dynamic behavior of the charge traps, we simulated an ensemble of 2000 independent charge trap dipole lattices, such as the one presented in Figure 8A, assuming that differences between the obtained net voltage, resulting from all the traps at distance $r \leq 1000 \mathrm{~nm}$, correspond to variability in potential barrier measured in experiments. ${ }^{86}$ In Figure $8 \mathrm{C}$ we show the resulting distribution of the potential values at the center of the graphene tunnel junction. The distribution can be fitted with a Gaussian function with the standard deviation $\sigma, \sigma e=\Delta \varphi=30 \mathrm{meV}$, and 
A
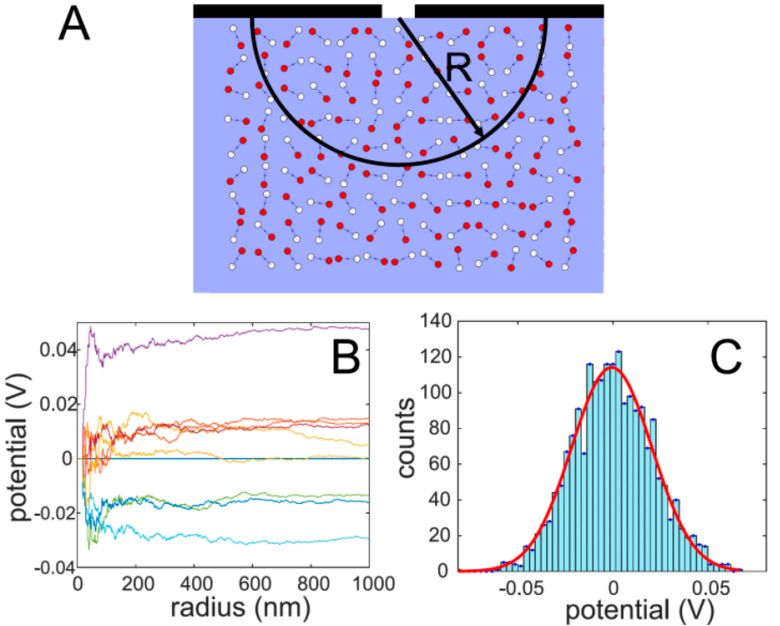

Figure 8. Potential shift due to random trap orientation. (A) Schematic diagram of a graphene tunnel junction on a dielectric substrate with embedded empty (white dots) and electron-filled charge traps (red dots). Pairs of charge traps creating electric dipoles are allowed to change randomly their orientation in each of the steps of the simulation, resulting in the change of electric potential in the center of the tunnel junction. (B) $V_{R}(R)$ dependence of cumulative potential of the tunnel junction generated by all the dipoles at distance $r<R$ as a function of distance $R$. The figure shows example traces obtained for nine different and independent charge trap distributions. The final potential value at $r<1000 \mathrm{~nm}$ is used for further analysis. (C) Distribution of the net potential of the graphene tunnel junction calculated for 2000 different charge trap dipole systems, with potential contributions summed for all of the traps at $r<1000 \mathrm{~nm}$. The distribution is fitted with a Gaussian function.

assuming a barrier height of $\varphi=500 \mathrm{meV}$ we obtain $\frac{\Delta \varphi}{\varphi}=0.06$. This value is in good agreement with the potential values obtained from the tight binding model $\mathrm{Ib} \frac{\Delta u}{u}=0.057$. Using eqs 1 and 2, we can now estimate the amplitude of current fluctuations. For a $1 \mathrm{~nm}$ wide tunnel junction, we find $\Delta I / I=0.4$. The parameters obtained in our numerical model are in good agreement with the tight binding model. The current ratio $\frac{\Delta I}{I}$ is also in accord with the distribution of the normalized noise amplitude at room temperature $\log \alpha=-3$ to 1 , as $\log (\Delta I / I)^{2}=-0.8$.

The estimated potential shifts are calculated assuming that there is a single point of junction sensitive to the electrostatic environment. Although tight binding models I and II are both capable of reproducing the main characteristics of current measurements at both cryogenic and room temperature (Figure 7$)$, comparing small tunneling distance $(1-2 \mathrm{~nm})$ to relatively large intertrap spacing $(\sim 10 \mathrm{~nm})$, we regard model I as more realistic.

Charge traps are distributed also over the entire graphenesubstrate interface, but only those traps located in the vicinity of the junction exert a sizable shift of the tunneling barrier. Traps located away from the junction, under the graphene leads, can still influence the conductance of the device by locally changing the density of states of carriers or their mobility. ${ }^{86,87}$ However, the effect of traps located under wider regions of graphene electrodes is limited, because these traps are not synchronized and switching of each of them gates only a small fragment of the graphene electrode, while there are many more parallel conduction paths. ${ }^{88}$ The same argument holds for fluctuations resulting from the electromigration of metal atoms at the gold-graphene interface: ${ }^{16,89}$ the contact resistance is only a fraction of resistance of the tunnel junction, such that the contribution of contact resistance fluctuations will be negligible. The large distance from the metal contacts to the tunnel junction $(2 \mu \mathrm{m})$ will also prevent metal atoms from migrating to the junction. Therefore, we conclude that the tunnel barrier in the junction remains the area of the device that is most sensitive to changes in the electrostatic environment. This highly localized sensitivity can be harnessed for molecular sensing applications. One example of high sensitivity of the investigated devices is the analysis of charge trap interactions in the vicinity of tunnel junction.

Charge Trap Interactions. Until now we have treated the RTSs as a purely stochastic process, with the independent dwell time values for consecutive current levels governed by Poisson statistics and random values of the switching current amplitude distributed according to a Gaussian distribution. However, it is known from single molecule measurements that the analysis of correlations in current values can reveal more details of a transport mechanism than a simple analysis of current traces. $^{90,91}$ The correlation in RTSs in a graphene tunnel junction is evident from correlation diagrams showing the amplitude of $n+1$ transition as a function of $n$ transition $\left(\Delta I_{n}, \Delta I_{n+1}\right)$. The RTS data takes the form of two main point clusters (Figure 9) corresponding to a down $\rightarrow$ up transition sequence $\left(\Delta I_{\text {down }}, \Delta I_{\text {up }}\right)$ (Figure $\left.9 \mathrm{~A}\right)$ and up $\rightarrow$ down
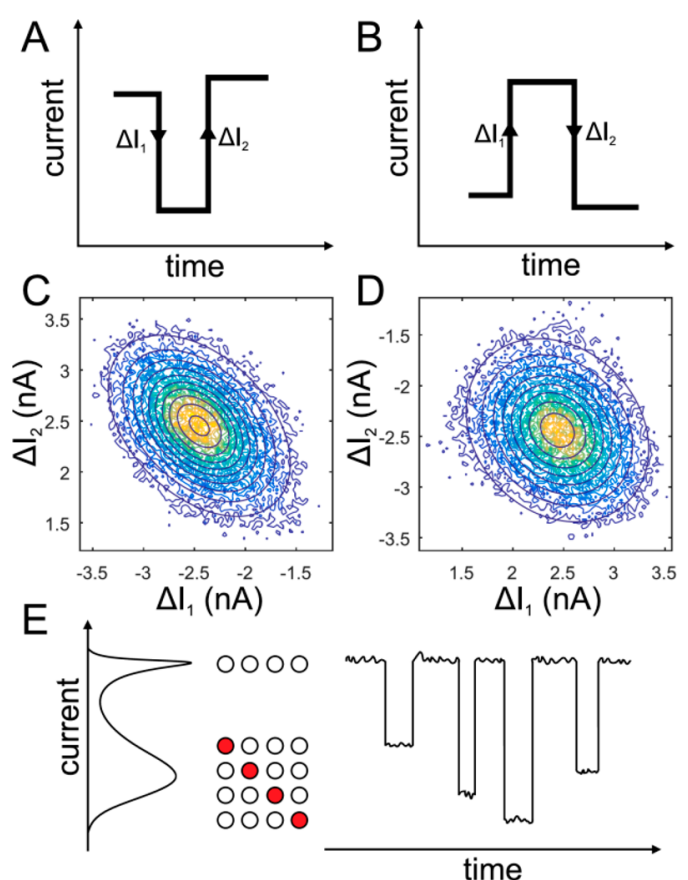

Figure 9. Correlation diagrams showing correlation between pairs of switching events $\left(\Delta I_{n}, \Delta I_{n+1}\right)$. Diagram of a pair of switching events (A) $\left(\Delta I_{\text {down }}, \Delta I_{\text {up }}\right)$ and (B) $\left(\Delta I_{\text {up }}, \Delta I_{\text {down }}\right)$. (C, D) Experimentally measured distributions of pairs of $\left(\Delta I_{1}, \Delta I_{2}\right)$ points with overlaid bivariate Gaussian distribution fits. There is higher asymmetry in the distribution of (C) $\left(\Delta I_{\text {down }}, \Delta I_{\text {up }}\right)$ events than of (D) $\left(\Delta I_{\text {up }}, \Delta I_{\text {down }}\right)$ events. (E) Diagram showing schematically how occupation of different empty traps (white dots) with a charge carrier (red dot) leads to the different current levels and results in the broadening of a current distribution for the low conductance state. 
$\left(\Delta I_{\text {up }}, \Delta I_{\text {down }}\right)$ sequence (Figure 9B). In the case of a single independent trap governing the transport the absolute values of the step amplitudes should be equal, $\left|\Delta I_{\text {up }}\right|=\left|\Delta I_{\text {down }}\right|$ resulting in symmetric circular distributions of points. There is, however, a sizable asymmetry in the $\left(\Delta I_{\text {down }}, \Delta I_{\text {up }}\right)$ distribution (Figure 9C) compared to the $\left(\Delta I_{\text {up }}, \Delta I_{\text {down }}\right)$ distribution (Figure 9D), which can be explained assuming that charge traps experience Coulomb interactions from their environment, that is other traps. ${ }^{40}$ If a trap is occupied, it prevents occupation of neighboring traps through Coulomb repulsion, however the neighboring traps might be energetically equivalent and thus any of them can be filled by a charge carrier. Occupation of different traps leads to a slightly different current level in the down state (Figure 9E). In contrast there is only one configuration for the up state, corresponding to the narrower distribution of possible current values. The asymmetry of the $\left(\Delta I_{n}, \Delta I_{n+1}\right)$ distribution can be reproduced by assuming the Gaussian distribution of the possible current values for both up and down states with the higher standard deviation of the latter distribution (SI).

\section{CONCLUSIONS}

We have demonstrated the presence of RTSs and a Lorentzian noise spectrum in graphene devices. The switching process leading to RTSs is not generated by the tunneling current, which serves only as a readout mechanism, as is evident from the constant relative current step amplitude $\Delta I / I \sim 0.1$. The capability of detecting single switching events shows high sensitivity of the graphene tunnel junctions to the local environment, which allows us to envisage highly sensitive graphene tunnel junction biosensors. The high sensitivity leads however to high noise levels.

The observed switching features can be explained by the gating of the tunnel barrier by charge carriers switching between oxide charge traps. Correlations in the amplitude of switching event pairs $\left(\Delta I_{n}, \Delta I_{n+1}\right)$ suggest the presence of Coulomb repulsion between traps, allowing only a single trap in the vicinity of the junction to be occupied.

At cryogenic temperatures only single traps are available, whereas at elevated temperatures more thermally excited traps can take part in switching. $I-t$ traces affected by these traps have a wandering line and log-normal current distribution due to the normal distribution of potential barrier heights. The superposition of Lorentzian spectra with different characteristic frequencies leads to the observation of $1 / f$ noise spectrum.

Our tight binding model reproduces qualitatively all the features of observed RTSs at cryogenic temperature and $1 / f$ noise at room temperature. The model assumes that the fluctuations are caused by the interaction of the quantum tunnel barrier with a classical environment. Our first model assumes that all quantum levels in the scattering region are driven collectively due to the averaged effect of all traps buried deeper in the oxide. Our second model assumes an individual interaction of the quantum levels in the scattering region with individual traps, which corresponds to the traps located close to the barrier. Both of the tight binding models lead to results which are consistent with the experimental measurements, indicating that in the measured graphene tunnel junctions both of the individual and collective models might be observed.

Our numerical model calculates the potential shift $\Delta \varphi$ and resulting current fluctuations amplitude $\Delta I$ due to the net effect of the traps in the substrate, assuming their constant density and dipole-type interactions. Agreement between the parameters related to current and potential shift obtained from experimental data, tight binding model, and numerical model supports attributing the noise in graphene tunnel junctions to charge traps.

\section{METHODS}

Fabrication of Graphene Devices. CVD-grown graphene, whose synthesis procedure has been previously described in ref 92 , is transferred into p-doped $\mathrm{Si}$ wafers with $300 \mathrm{~nm} \mathrm{SiO}$ layer and patterned $10 \mathrm{~nm} \mathrm{Cr} / 70 \mathrm{~nm}$ Au electrodes. Graphene is patterned into $200 \mathrm{~nm}$ wide constrictions using a combination of electron-beam lithography (JEOL 5500FS) with a negative resist ma-N 2405 and oxygen plasma etching.

Electroburning of Tunnel Junctions. Devices are contacted using automated probe station. The formation of tunnel junctions is achieved by feedback-controlled electroburning of graphene constrictions. Electroburning relies on the application of bias to the constriction with the simultaneous measurement of current (Figure SI8A for electroburning traces). The bias is increased at low constant rate of $750 \mathrm{mV} \mathrm{s}^{-1}$ resulting in initial linear increase of the current; at some point further increase of the voltage leads to the decrease of the slope of $I-V$ curve and consequent decrease of current. This point marks the onset of electroburning due to the removal of carbon atoms caused by the high temperature in the constriction due to the joule heating. Once the current drop is detected the feedback loop decreases the voltage to zero at a high rate of $225 \mathrm{~V} \mathrm{~s}^{-1}$ to prevent the uncontrolled breakdown of the constriction. This electroburning cycle is repeated multiple times for each device, with increased resistance after each iteration, verified by the $I-V$ measurement. The process is stopped at $500 \mathrm{M} \Omega$ resistance, which corresponds to the formation of a tunnel junction. The tunneling regime is confirmed by the measurement of a nonlinear $I-V$ (Figure SI8B).

Determination of Tunnelling Distance. The nonlinear $I-V$ curves obtained for successfully burned graphene devices are subsequently used to estimate the tunneling distance, which is achieved by fitting the $I-V$ curves to a nonlinear Simmons model, assuming tunneling process through an asymmetric potential barrier. ${ }^{1,63}$ The fitting model is implemented in a form of iterative script which calculates current values for given voltage range, using as fitting parameters the width, height, and asymmetry factor of the potential barrier, with tunneling barrier width corresponding to the size of tunnel gap. Details of the implementation of fitting with Simmons model are given in the SI, as well as statistical distribution of fitted tunneling gap widths and estimation of the fitting error. An example of measured $I-V$ curve and fitted Simons curve is also presented in Figure SI8B.

Electric Measurements. Devices with features of tunneling current and the tunneling distance obtained from the Simmons fit on the order of 1-2 $\mathrm{nm}$ were used for further measurements. Devices were measured in a custom-built cryogenic liquid dipper, which was vacuum pumped to the pressure of $10^{-4}$ mbar and dipped in liquid nitrogen to obtain temperature of $77 \mathrm{~K}$. Devices at room temperature were measured both in vacuum and ambient atmosphere, without any difference in the current signal or noise. Room temperature measurements were also performed in the same dipper, which also screens external electric fields. All measured devices were connected to Axopatch $200 \mathrm{~B}$ voltage clamp amplifier which offers unrivalled noise performance among other commercial discrete electronic measurement systems. ${ }^{93}$ The graphene devices were connected through the Axopatch headstage preamplifier, which was kept in a Faraday box to minimize the external noise contributions. The length of wires connecting the head stage and dipper was kept to minimum $(\sim 10-20 \mathrm{~cm})$ to minimize the noise pick-up and capacitance of the wires. The Axopatch 200B was operated in a voltage clamp mode and was used to bias the devices. The measured current was recorded and applied voltage controlled through Digidata $1440 \mathrm{~A}$ acquisition card. A Bessel filter with 1 or $10 \mathrm{kHz}$ filter frequency was applied to the signal and current was sampled at $100 \mathrm{kHz}$ frequency. Noise spectra were calculated on the basis of Fourier transform of $I-t$ traces. Recorded 
traces were divided into 10 sections, and noise spectrum was calculated for each of the sections individually. The spectra shown in this Article are an average of 10 noise spectra. In order to characterize the intrinsic noise level of the measurement system and prevent any instrumentation artifact, we characterized also open circuit noise level and thermal noise recorded in resistors (Figure SI15)

\section{ASSOCIATED CONTENT}

\section{S Supporting Information}

The Supporting Information is available free of charge on the ACS Publications website at DOI: 10.1021/acsnano.8b04713.

Details of the classical fluctuator model used in the tight binding models; voltage and current scaling behavior of tight binding model I; results for the tight binding model II; electroburning and tunneling $I-V$ traces for graphene tunnel junctions; statistical distribution of width of electroburnt devices and estimation of the gap width fitting error; simulated $\left(\Delta I_{n}, \Delta I_{n+1}\right)$ cluster asymmetry; long-term stability of RTSs; further details on the electrostatic shift of tunneling $I-V$ due to charge traps; distribution of $1 / f$ noise $\gamma$ slopes as a function of tunneling current; noise characterization of the measurement system, including open-circuit and thermal noise measurements (PDF)

\section{AUTHOR INFORMATION}

\section{Corresponding Author}

*E-mail: jan.mol@materials.ox.ac.uk.

ORCID

Paweł Puczkarski: 0000-0001-5477-1837

Hatef Sadeghi: 0000-0001-5398-8620

Yuewen Sheng: 0000-0003-3067-9520

Jamie H. Warner: 0000-0002-1271-2019

Colin J. Lambert: 0000-0003-2332-9610

Jan A. Mol: 0000-0003-0411-2598

\section{Author Contributions}

P.P. and A.K. performed the measurements. P.P. analyzed experimental data and estimated potential distributions. Q.W. and S.H. carried out the tight binding simulations. H.S. guided the simulations. C.J.L. conceptualized the tight binding noisedriven model. Y.S. and J.H.W. synthesized graphene. C.J.L., G.A.D.B., and J.A.M. supervised the project. All contributed to the interpretation of results and writing of the paper.

\section{Notes}

The authors declare no competing financial interest.

\section{ACKNOWLEDGMENTS}

This work was supported by the UK EPSRC (EP/N509711/1, EP/N017188/1, and EP/R029229/1). J.A.M. acknowledges a RAEng Research Fellowship. H.S. acknowledges a Leverhulme Early Career Fellowship No. ECF-2017-186. We thank J. L. Swett for taking SEM images.

\section{REFERENCES}

(1) Prins, F.; Barreiro, A.; Ruitenberg, J. W.; Seldenthuis, J. S.; Aliaga-Alcalde, N.; Vandersypen, L. M. K.; Van Der Zant, H. S. J. Room-Temperature Gating of Molecular Junctions Using Few-Layer Graphene Nanogap Electrodes. Nano Lett. 2011, 11, 4607-4611.

(2) Mol, J. A.; Lau, C. S.; Lewis, W. J. M.; Sadeghi, H.; Roche, C.; Cnossen, A.; Warner, J. H.; Lambert, C. J.; Anderson, H. L.; Briggs, G. A. D. Graphene-Porphyrin Single-Molecule Transistors. Nanoscale 2015, 7, 13181-13185.
(3) Sadeghi, H.; Sangtarash, S.; Lambert, C. J. Robust Molecular Anchoring to Graphene Electrodes. Nano Lett. 2017, 17, 4611-4618.

(4) Jia, C.; Migliore, A.; Xin, N.; Huang, S.; Wang, J.; Yang, Q.; Wang, S.; Chen, H.; Wang, D.; Feng, B.; Liu, Z.; Zhang, G.; Qu, D. H.; Tian, H.; Ratner, M. A.; Xu, H. Q.; Nitzan, A.; Guo, X. Covalently Bonded Single-Molecule Junctions with Stable and Reversible Photoswitched Conductivity. Science 2016, 352, 1443-1446.

(5) Zhou, C.; Li, X.; Gong, Z.; Jia, C.; Lin, Y.; Gu, C.; He, G.; Zhong, Y.; Yang, J.; Guo, X. Direct Observation of Single-Molecule Hydrogen-Bond Dynamics with Single-Bond Resolution. Nat. Commun. 2018, 9, 807.

(6) Wen, H.; Li, W.; Chen, J.; He, G.; Li, L.; Olson, M. A.; Sue, A. C.; Stoddart, J. F.; Guo, X. Complex Formation Dynamics in a SingleMolecule Electronic Device. Sci. Adv. 2016, 2, e1601113.

(7) Xu, Q.; Scuri, G.; Mathewson, C.; Kim, P.; Nuckolls, C.; Bouilly, D. Single Electron Transistor with Single Aromatic Ring Molecule Covalently Connected to Graphene Nanogaps. Nano Lett. 2017, 17, 5335-5341.

(8) Sadeghi, H.; Mol, J. A.; Lau, C. S.; Briggs, G. A. D.; Warner, J.; Lambert, C. J. Conductance Enlargement in Picoscale Electroburnt Graphene Nanojunctions. Proc. Natl. Acad. Sci. U. S. A. 2015, 112, 2658-2663.

(9) Postma, H. W. Ch. Rapid Sequencing of Individual DNA Molecules in Graphene Nanogaps. Nano Lett. 2010, 10, 420-425.

(10) Geim, A. K.; Novoselov, K. S. The Rise of Graphene. Nat. Mater. 2007, 6, 183-191.

(11) Balandin, A. A. Low-Frequency $1 / f$ Noise in Graphene Devices. Nat. Nanotechnol. 2013, 8, 549-555.

(12) Takeshita, S.; Matsuo, S.; Tanaka, T.; Nakaharai, S.; Tsukagoshi, K.; Moriyama, T.; Ono, T.; Arakawa, T.; Kobayashi, K. Anomalous Behavior of $1 / f$ Noise in Graphene near the Charge Neutrality Point. Appl. Phys. Lett. 2016, 108, 103106.

(13) Liu, G.; Rumyantsev, S.; Shur, M. S.; Balandin, A. A. Origin of $1 / \mathrm{f}$ Noise in Graphene Multilayers: Surface vs. Volume. Appl. Phys. Lett. 2013, 102, 093111.

(14) Kaverzin, A. A.; Mayorov, A. S.; Shytov, A.; Horsell, D. W. Impurities as a Source of $1 / \mathrm{f}$ Noise in Graphene. Phys. Rev. B: Condens. Matter Mater. Phys. 2012, 85, 075435.

(15) Pal, A. N.; Ghatak, S.; Kochat, V.; Sneha, E. S.; Sampathkumar, A.; Raghavan, S.; Ghosh, A. Microscopic Mechanism of $1 / f$ Noise in Graphene: Role of Energy Band Dispersion. ACS Nano 2011, 5, 2075-2081.

(16) Liu, G.; Rumyantsev, S.; Shur, M.; Balandin, A. A. Graphene Thickness-Graded Transistors with Reduced Electronic Noise. Appl. Phys. Lett. 2012, 100, 033103.

(17) Zhang, Y.; Mendez, E. E.; Du, X. Mobility-Dependent LowFrequency Noise in Graphene Field-Effect Transistors. ACS Nano 2011, 5, 8124-8130.

(18) Zahid Hossain, M.; Rumyantsev, S.; Shur, M. S.; Balandin, A. A. Reduction of $1 / \mathrm{f}$ Noise in Graphene after Electron-Beam Irradiation. Appl. Phys. Lett. 2013, 102, 153512.

(19) Rumyantsev, S.; Liu, G.; Stillman, W.; Shur, M.; Balandin, A. A. Electrical and Noise Characteristics of Graphene Field-Effect Transistors: Ambient Effects, Noise Sources and Physical Mechanisms. J. Phys.: Condens. Matter 2010, 22, 395302.

(20) Heller, I.; Chatoor, S.; Mannik, J.; Zevenbergen, M. A. G.; Oostinga, J. B.; Morpurgo, A. F.; Dekker, C.; Lemay, S. G. Charge Noise in Graphene Transistors. Nano Lett. 2010, 10, 1563-1567.

(21) Sun, N.; Tahy, K.; Xing, H.; Jena, D.; Arnold, G.; Ruggiero, S. T. Electrical Noise and Transport Properties of Graphene. J. Low Temp. Phys. 2013, 172, 202-211.

(22) Lee, S. K.; Kang, C. G.; Lee, Y. G.; Cho, C.; Park, E.; Chung, H. J.; Seo, S.; Lee, H. D.; Lee, B. H. Correlation of Low Frequency Noise Characteristics with the Interfacial Charge Exchange Reaction at Graphene Devices. Carbon 2012, 50, 4046-4051.

(23) Cheng, Z.; Li, Q.; Li, Z.; Zhou, Q.; Fang, Y. Suspended Graphene Sensors with Improved Signal and Reduced Noise. Nano Lett. 2010, 10, 1864-1868. 
(24) Sung, M. G.; Lee, H.; Heo, K.; Byun, K.-E. E.; Kim, T.; Seo, D. H.; Seo, S.; Hong, S. Scanning Noise Microscopy on Graphene Devices. ACS Nano 2011, 5, 8620-8628.

(25) Kumar, M.; Laitinen, A.; Cox, D.; Hakonen, P. J. Ultra Low $1 / f$ Noise in Suspended Bilayer Graphene. Appl. Phys. Lett. 2015, 106, 263505.

(26) Pal, A. N.; Ghosh, A. Ultralow Noise Field-Effect Transistor from Multilayer Graphene. Appl. Phys. Lett. 2009, 95, 082105.

(27) Liu, G.; Stillman, W.; Rumyantsev, S.; Shao, Q.; Shur, M.; Balandin, A. A. Low-Frequency Electronic Noise in the Double-Gate Single-Layer Graphene Transistors. Appl. Phys. Lett. 2009, 95, 033103.

(28) Pal, A. N.; Ghosh, A. Resistance Noise in Electrically Biased Bilayer Graphene. Phys. Rev. Lett. 2009, 102, 126805.

(29) Lin, Y. M.; Avouris, P. Strong Suppression of Electrical Noise in Bilayer Graphene Nanodevices. Nano Lett. 2008, 8, 2119-2125.

(30) Pedersen, J. N.; Boynton, P.; Ventra, M. Di; Jauho, A.-P.; Flyvbjerg, H. Classification of DNA Nucleotides with Transverse Tunneling Currents. Nanotechnology 2017, 28, 015502.

(31) Lagerqvist, J.; Zwolak, M.; Di Ventra, M. Influence of the Environment and Probes on Rapid DNA Sequencing via Transverse Electronic Transport. Biophys. J. 2007, 93, 2384-2390.

(32) Krems, M.; Zwolak, M.; Pershin, Y. V.; Di Ventra, M. Effect of Noise on DNA Sequencing via Transverse Electronic Transport. Biophys. J. 2009, 97, 1990-1996.

(33) Sadeghi, H.; Algaragholy, L.; Pope, T.; Bailey, S.; Visontai, D.; Manrique, D.; Ferrer, J.; Garcia-Suarez, V.; Sangtarash, S.; Lambert, C. J. Graphene Sculpturene Nanopores for DNA Nucleobase Sensing. J. Phys. Chem. B 2014, 118, 6908-6914.

(34) Sadeghi, H.; Bailey, S.; Lambert, C. J. Silicene-Based DNA Nucleobase Sensing. Appl. Phys. Lett. 2014, 104, 103104.

(35) Weissman, M. B. $1 / f$ Noise and Other Slow, Nonexponential Kinetics in Condensed Matter. Rev. Mod. Phys. 1988, 60, 537-571.

(36) Kirton, M. J.; Uren, M. J. Noise in Solid-State Microstructures: A New Perspective on Individual Defects, Interface States and LowFrequency $(1 / f)$ Noise. Adv. Phys. 1989, 38, 367-468.

(37) Ralls, S. K.; Skocpol, W. J.; Jackel, L. D.; Howard, R. E.; Fetter, L. A.; Epworth, R. W.; Tennant, D. M. Discrete Resistance Switching in Submicrometer Silicon Inversion Layers: Individual Interface Traps and Low-Frequency (1/f?) Noise. Phys. Rev. Lett. 1984, 52, 228-231.

(38) Kandiah, K.; Deighton, M. O.; Whiting, F. B. A Physical Model for Random Telegraph Signal Currents in Semiconductor Devices. J. Appl. Phys. 1989, 66, 937-948.

(39) Dutta, P.; Horn, P. M. Low-Frequency Fluctuations in Solids: 1/f Noise. Rev. Mod. Phys. 1981, 53, 497-516.

(40) Rogers, C.; Buhrman, R.; Gallagher, W.; Raider, S.; Kleinsasser, A.; Sandstrom, R. Electron Trap States and Low Frequency Noise in Tunnel Junctions. IEEE Trans. Magn. 1987, 23, 1658-1661.

(41) Kohlstedt, H.; Gundlach, K. H.; Kuriki, S. Electric Forming and Telegraph Noise in Tunnel Junctions. J. Appl. Phys. 1993, 73, 25642568.

(42) Liefrink, F.; Scholten, A. J.; Dekker, C. Low-Frequency Noise of Quantum Point Contacts in the Ballistic and Quantum Hall Regime. Phys. B 1991, 175, 213-216.

(43) Li, Y. P.; Tsui, D. C.; Heremans, J. J.; Simmons, J. A.; Weimann, G. W. Low-Frequency Noise in Transport through Quantum Point Contacts. Appl. Phys. Lett. 1990, 57, 774-776.

(44) Smeets, R. M. M.; Keyser, U. F.; Wu, M. Y.; Dekker, N. H.; Dekker, C. Nanobubbles in Solid-State Nanopores. Phys. Rev. Lett. 2006, 97, 088101.

(45) Wen, C.; Zeng, S.; Arstila, K.; Sajavaara, T.; Zhu, Y.; Zhang, Z.; Zhang, S.-L. Generalized Noise Study of Solid-State Nanopores at Low Frequencies. ACS Sensors 2017, 2, 300-307.

(46) Smeets, R. M. M.; Dekker, N. H.; Dekker, C. Low-Frequency Noise in Solid-State Nanopores. Nanotechnology 2009, 20, 095501.

(47) Siwy, Z.; Fuliński, A. Origin of $1 / f^{x}$ Noise in Membrane Channel Currents. Phys. Rev. Lett. 2002, 89, 158101.

(48) Heerema, S. J.; Schneider, G. F.; Rozemuller, M.; Vicarelli, L.; Zandbergen, H. W.; Dekker, C. $1 / f$ Noise in Graphene Nanopores. Nanotechnology 2015, 26, 074001.
(49) Smeets, R. M. M.; Keyser, U. F.; Dekker, N. H.; Dekker, C. Noise in solid-state nanopores. Proc. Natl. Acad. Sci. U. S. A. 2008, 105, 417-421.

(50) Costanzi, B. N.; Dahlberg, E. D. Emergent $1 / f$ Noise in Ensembles of Random Telegraph Noise Oscillator. Phys. Rev. Lett. 2017, 119, 097201.

(51) McWhorter, A. L.; Kingston, R. H. Semiconductor Surface Physics; University of Pennsylvania Press, 1957.

(52) Xu, G.; Zhang, Y.; Duan, X.; Balandin, A. A.; Wang, K. L. Variability Effects in Graphene: Challenges and Opportunities for Device Engineering and Applications. Proc. IEEE 2013, 101, 16701688.

(53) Liu, F.; Bao, M.; Kim, H. J.; Wang, K. L.; Li, C.; Liu, X.; Zhou, C. Giant Random Telegraph Signals in the Carbon Nanotubes as a Single Defect Probe. Appl. Phys. Lett. 2005, 86, 163102.

(54) Xu, G.; Torres, C. M.; Song, E. B.; Tang, J.; Bai, J.; Duan, X.; Zhang, Y.; Wang, K. L. Enhanced Conductance Fluctuation by Quantum Confinement Effect in Graphene Nanoribbons. Nano Lett. 2010, 10, 4590-4594.

(55) Arielly, R.; Vadai, M.; Kardash, D.; Noy, G.; Selzer, Y. RealTime Detection of Redox Events in Molecular Junctions. J. Am. Chem. Soc. 2014, 136, 2674-2680.

(56) Vardi, Y.; Guttman, A.; Bar-Joseph, I. Random Telegraph Signal in a Metallic Double-Dot System. Nano Lett. 2014, 14, 2794-2799.

(57) Kim, Y.; Song, H.; Kim, D.; Lee, T.; Jeong, H. Noise Characteristics of Charge Tunneling via Localized States in MetalMolecule-Metal Junctions. ACS Nano 2010, 4, 4426-4430.

(58) Stroscio, J. A.; Celotta, R. J. Controlling the Dynamics of a Single Atom in Lateral Atom Manipulation. Science 2004, 306, 242247.

(59) Sperl, A.; Kröger, J.; Berndt, R. Direct Observation of Conductance Fluctuations of a Single-Atom Tunneling Contact. Phys. Rev. B: Condens. Matter Mater. Phys. 2010, 81, 035406.

(60) Klein, H.; Leoni, T.; Zoubkoff, R.; Dumas, P.; Saul, A. Conductance Fluctuations in Gold Point Contacts: An Atomistic Picture. Nanotechnology 2012, 23, 235707.

(61) Tsutsui, M.; Kurokawa, S.; Sakai, A. Bias-Induced Local Heating in Atom-Sized Metal Contacts at 77 K. Appl. Phys. Lett. 2007, 90, 133121.

(62) Liefrink, F.; Dijkhuis, J. I.; van Houten, H. Low-Frequency Noise in Quantum Point Contacts. Semicond. Sci. Technol. 1994, 9, $2178-2189$.

(63) Simmons, J. G. Generalized Formula for the Electric Tunnel Effect between Similar Electrodes Separated by a Thin Insulating Film. J. Appl. Phys. 1963, 34, 1793-1803.

(64) Lau, C. S.; Mol, J. A.; Warner, J. H.; Briggs, G. A. D. Nanoscale Control of Graphene Electrodes. Phys. Chem. Chem. Phys. 2014, 16, 20398-20401.

(65) Nef, C.; Posa, L.; Makk, P.; Fu, W.; Halbritter, A.; Schonenberger, C.; Calame, M. High-Yield Fabrication of Nm-Size Gaps in Monolayer CVD Graphene. Nanoscale 2014, 6, 7249-7254.

(66) Machlup, S. Noise in Semiconductors: Spectrum of a TwoParameter Random Signal. J. Appl. Phys. 1954, 25, 341-343.

(67) Balandin, A. A. Noise and Fluctuations Control in Electronic Devices; American Scientific Publishers: Los Angeles, 2002.

(68) Yuzhelevski, Y.; Yuzhelevski, M.; Jung, G. Random Telegraph Noise Analysis in Time Domain. Rev. Sci. Instrum. 2000, 71, 1681.

(69) Yeh, S.; Chang, W.; Lin, J. Probing Nanocrystalline Grain Dynamics in Nanodevices. Sci. Adv. 2017, 3, e1700135.

(70) Uren, M. J.; Kirton, M. J.; Collins, S. Anomalous Telegraph Noise in Small-Area Silicon Metal-Oxide-Semiconductor Field-Effect Transistors. Phys. Rev. B: Condens. Matter Mater. Phys. 1988, 37, $8346-8350$

(71) Stolyarov, M. A.; Liu, G.; Rumyantsev, S. L.; Shur, M.; Balandin, A. A. Suppression of $1 / f$ Noise in near-Ballistic h-BNGraphene-h-BN Heterostructure Field-Effect Transistors. Appl. Phys. Lett. 2015, 107, 023106. 
(72) Sun, N.; Tahy, K.; Xing, H.; Jena, D.; Arnold, G.; Ruggiero, S. T. Electrical Noise and Transport Properties of Graphene. J. Low Temp. Phys. 2013, 172, 202-211.

(73) Smeets, R. M. M.; Keyser, U. F.; Dekker, N. H.; Dekker, C. Noise in Solid-State Nanopores. Proc. Natl. Acad. Sci. U. S. A. 2008, $105,417-421$.

(74) Lim, Y. F.; Xiong, Y. Z.; Singh, N.; Yang, R.; Jiang, Y.; Chan, D. S. H.; Loh, W. Y.; Bera, L. K.; Lo, G. Q.; Balasubramanian, N.; Kwong, D. L. Random Telegraph Signal Noise in Gate-All-around SiFinFET with Ultranarrow Body. IEEE Electron Device Lett. 2006, 27, $765-768$.

(75) Zhuge, J.; Wang, R.; Huang, R.; Tian, Y.; Zhang, L.; Kim, D.W.; Park, D.; Wang, Y. Investigation of Low-Frequency Noise in Slicon Nanowire MOSFETs. IEEE Electron Device Lett. 2009, 30, 5760.

(76) Clément, N.; Larrieu, G.; Dubois, E. Low-Frequency Noise in Schottky-Barrier-Based Nanoscale Field-Effect Transistors. IEEE Trans. Electron Devices 2012, 59, 180-187.

(77) Magnone, P.; Crupi, F.; Giusi, G.; Pace, C.; Simoen, E.; Claeys, C.; Pantisano, L.; Maji, D.; Rao, V. R.; Srinivasan, P. Noise in Drain and Gate Current of MOSFETs With High- Gate Stacks. IEEE Trans. Device Mater. Reliab. 2009, 9, 180-189.

(78) Theodorou, C. G.; Fasarakis, N.; Hoffman, T.; Chiarella, T.; Ghibaudo, G.; Dimitriadis, C. A. Flicker Noise in N-Channel Nanoscale Tri-Gate Fin-Shaped Field-Effect Transistors. Appl. Phys. Lett. 2012, 101, 243512.

(79) Lee, J. S.; Choi, Y. K.; Ha, D.; Balasubramanian, S.; King, T. J.; Bokor, J. Hydrogen Annealing Effect on DC and Low-Frequency Noise Characteristics in CMOS FinFETs. IEEE Electron Device Lett. 2003, 24, 186-188.

(80) Lin, Y.; Appenzeller, J.; Knoch, J.; Chen, Z.; Avouris, P. LowFrequency Current Fluctuations in Individual Semiconducting SingleWall Carbon Nanotubes. Nano Lett. 2006, 6, 930-936.

(81) Ishigami, M.; Chen, J. H.; Williams, E. D.; Tobias, D.; Chen, Y. F.; Fuhrer, M. S. Hooge's Constant for Carbon Nanotube Field Effect Transistors. Appl. Phys. Lett. 2006, 88, 203116.

(82) Tarkiainen, R.; Roschier, L.; Ahlskog, M.; Paalanen, M.; Hakonen, P. Low-Frequency Current Noise and Resistance Fluctuations in Multiwalled Carbon Nanotubes. Phys. E 2005, 28, $57-65$.

(83) Heller, I.; Mannik, J.; Lemay, S. G.; Dekker, C. Optimizing the Signal-to-Noise Ratio for Biosensing with Carbon Nanotube Transistors. Nano Lett. 2009, 9, 377-382.

(84) Tersoff, J. Low-Frequency Noise in Nanoscale Ballistic Transistors. Nano Lett. 2007, 7, 194-198.

(85) Maneglia, Y.; Bauza, D. Extraction of Slow Oxide Trap Concentration Profiles in Metal-oxide-semiconductor Transistors Using the Charge Pumping Method. J. Appl. Phys. 1996, 79, 41874192.

(86) Liu, B.; Akis, R.; Ferry, D. K.; Bohra, G.; Somphonsane, R.; Ramamoorthy, H.; Bird, J. P. Conductance Fluctuations in Graphene in the Presence of Long-Range Disorder. J. Phys.: Condens. Matter 2016, 28, 135302.

(87) Li, W.; Chen, X.; Wang, L.; He, Y.; Wu, Z.; Cai, Y.; Zhang, M.; Wang, Y.; Han, Y.; Lortz, R. W.; Zhang, Z. Q.; Sheng, P.; Wang, N. Density of States and Its Local Fluctuations Determined by Capacitance of Strongly Disordered Graphene. Sci. Rep. 2013, 3, 1772.

(88) Lee, H.; Cho, D.; Shekhar, S.; Kim, J.; Park, J.; Hong, B. H.; Hong, S. Nanoscale Direct Mapping of Noise Source Activities on Graphene Domains. ACS Nano 2016, 10, 10135-10142.

(89) Shao, Q.; Liu, G.; Teweldebrhan, D.; Balandin, A. a.; Rumyantsev, S.; Shur, M. S.; Yan, D. Flicker Noise in Bilayer Graphene Transistors. IEEE Electron Device Lett. 2009, 30, 288-290.

(90) Makk, P.; Tomaszewski, D.; Martinek, J.; Balogh, Z.; Csonka, S.; Wawrzyniak, M.; Frei, M.; Venkataraman, L.; Halbritter, A. Correlation Analysis of Atomic and Single-Molecule Junction Conductance. ACS Nano 2012, 6, 3411-3423.
(91) Bellisario, D. O.; Liu, A. T.; Kozawa, D.; Han, R.; Harris, J. K.; Zabala, R. B.; Wang, Q. H.; Agrawal, K. V.; Son, Y.; Strano, M. S. Experimental Observation of Real Time Molecular Dynamics Using Electromigrated Tunnel Junctions. J. Phys. Chem. C 2017, 121, 22550-22558.

(92) Sheng, Y.; Rong, Y.; He, Z.; Fan, Y.; Warner, J. H. Uniformity of Large-Area Bilayer Graphene Grown by Chemical Vapor Deposition. Nanotechnology 2015, 26, 395601.

(93) Rosenstein, J. K.; Wanunu, M.; Merchant, C. A.; Drndic, M.; Shepard, K. L. Integrated Nanopore Sensing Platform with SubMicrosecond Temporal Resolution. Nat. Methods 2012, 9, 487-494. 